\title{
Pensamiento crítico y adquisición de la competencia estratégica en estudiantes de traducción
}

\author{
SOFÍA LÉVANO CASTRO* \\ Universidad Ricardo Palma - Perú \\ Recibido el 04-09-18; primera evaluación el 10-12-19; \\ segunda evaluación el 21-01-20; aceptado el 10-02-20
}

\section{RESUMEN}

Este estudio tiene como objetivo demostrar la influencia de un programa de intervención para el desarrollo del pensamiento crítico en la adquisición de la competencia estratégica de los estudiantes de traducción de una universidad de Lima. Para ello, se realizó una investigación con diseño cuasiexperimental de preprueba y posprueba; con grupo experimental y de control. El objeto de estudio lo constituyó un programa de intervención para el desarrollo del pensamiento crítico aplicado al grupo experimental. Los resultados demostraron que las actividades diseñadas para el desarrollo del pensamiento crítico influyeron significativamente en la competencia estratégica, que se midió a través de la capacidad de analizar problemas, evaluar traducciones y autoevaluar las traducciones propias.

Palabras clave: pensamiento crítico, competencia estratégica, solución de problemas, evaluación de traducciones.

\section{Critical Thinking and Strategic Competence Acquisition in Translation Students}

\section{Abstract}

This paper aims to demonstrate the influence of an intervention program for the development of critical thinking on the acquisition of strategic competence of translation students in a university in Lima. For this purpose, a quasi-experimental research with pre-test and post-test design with control and experimental groups was carried out. The object of study was an intervention program for the development of critical thinking applied to the experimental group. The findings

\footnotetext{
* Docente de la especialidad de Traducción e Interpretación y de la maestría en Traducción de la Universidad Ricardo Palma, Perú. Licenciada en Traducción. Maestra en Docencia Superior por la Universidad Ricardo Palma. Doctora en Psicología Educacional y Tutorial por la Universidad Enrique Guzmán y Valle. Correo electrónico: sofia.levano@urp.edu.pe
} 
showed that the activities designed for the development of critical thinking significantly influenced strategic competence, which was measured through the student's ability to analyze problems, evaluate translations and assess their own translations.

Keywords: critical thinking, strategic competence, problem solving, translation quality assessment.

Pensamento crítico e aquisição de competência estratégica em estudantes de tradução

\section{Resumo}

O objetivo deste estudo é demonstrar a influência de um programa de intervenção para o desenvolvimento do pensamento crítico na aquisição da competência estratégica de estudantes de tradução em uma universidade em Lima. Para tanto, foi realizada uma pesquisa com delineamento quase-experimental pré-teste e pós-teste; com grupo experimental e controle. $\mathrm{O}$ objeto de estudo foi um programa de intervenção para o desenvolvimento do pensamento crítico aplicado ao grupo experimental. Os resultados mostraram que as atividades projetadas para o desenvolvimento do pensamento crítico influenciaram significativamente a competência estratégica, a qual foi medida pela capacidade de analisar problemas, avaliar traduçóes e autoavaliar as traduçóes.

Palavras-chave: pensamento crítico, competência estratégica, resolução de problemas, avaliação de tradução 


\section{INTRODUCCIÓN}

La tecnologización y globalización del aprendizaje y la automatización de la traducción exigen que el docente de traducción propicie el desarrollo intelectual de los estudiantes para que puedan comprender diversas formas de pensamiento y expresiones culturales, y luego transferirlas adecuadamente a otra lengua. En la sociedad de la información, la transferencia de conocimiento requiere de habilidades de pensamiento crítico. Morin (1999) hace referencia a la necesidad de desarrollar un pensamiento complejo en lugar de un pensamiento simple que deje de lado las fragmentaciones de los saberes y busque de forma solidaria y transdisciplinaria soluciones creativas a los problemas transversales, transnacionales y multidimensionales. Para ello, se requiere formar traductores que gestionen la información de forma analítica, ubiquen información relevante en sus lenguas de trabajo, la organicen y la apliquen para solucionar problemas lingüísticos y culturales.

Se han realizado varias investigaciones sobre pensamiento crítico en diferentes campos. Betancourth, Enríquez y Castillo (2013) determinaron los efectos de un programa de intervención para desarrollar el pensamiento crítico basado en la controversia socrática en estudiantes de psicología. El enfoque fue cuantitativo con un diseño cuasiexperimental con preprueba y posprueba. Los resultados indican que la controversia socrática fomenta la capacidad para formular hipótesis, usar argumentos basados en la experiencia, autoevaluar el propio razonamiento y reconocer opiniones diferentes. Reupo (2015) estudió el impacto de una propuesta constructivista que incorporaba el uso de TIC en estudiantes de ingeniería de sistemas de la Universidad Nacional Pedro Ruiz Gallo de Lambayeque. La metodología fue cuasiexperimental con un solo grupo de estudio. Los resultados mostraron que la ejercitación en resolver problemas, formular pregunta y el uso de las TIC contribuyó en el desarrollo de habilidades del pensamiento. Pineda y Cerrón (2015) realizaron una investigación para determinar la relación entre el pensamiento crítico y el rendimiento académico de los estudiantes de Lenguas, Literatura y Comunicación de la Facultad de Educación de la Universidad Nacional del Centro del Perú-Huancayo en el año 2013. Para ello, realizaron un estudio cuantitativo aplicado. La muestra poblacional estuvo compuesta por 107 estudiantes. Las conclusiones del estudio indican que el pensamiento crítico y el rendimiento académico están estrechamente relacionados.

La traducción es una actividad compleja en la que intervienen operaciones cognitivas de orden superior que permiten captar el sentido del texto original y expresarlo de forma natural, adecuada y aceptable para el receptor del texto meta. 
La traducción no es solo una actividad lingüística, sino cognitiva vinculada con el procesamiento de la información. En la carrera de Traducción e Interpretación de la Universidad Ricardo Palma, el entrenamiento en traducción se inicia en el sexto ciclo, luego de culminar la formación en idiomas y cursos teóricos. Por lo tanto, la formación del traductor, desde su fase inicial, debe orientarse al desarrollo de la capacidad de análisis, síntesis y evaluación. Hemos observado que los alumnos del primer taller de traducción tienden a la traducción literal en un inicio a pesar de conocer los fundamentos teóricos de la traducción interpretativa o traducción del sentido. La captación del sentido es la síntesis del proceso de comprensión y el resultado de la abstracción entre la información que proporciona el texto y los saberes previos. El traductor como pensador crítico debe analizar la información de entrada y la que produce en otra lengua, y, para ello, es necesario que cuestione su propio entendimiento $y$ busque en el texto pistas que permitan confirmar predicciones en cuanto al sentido del texto.

En una época en la que la inteligencia artificial se desarrolla vertiginosamente, en la que prolifera una fe ciega por la tecnología y en la que la demanda de traducciones rápidas condiciona la labor del traductor, es necesario formar traductores estratégicos y eficaces que puedan hacer frente a los avatares del mercado profesional sin descuidar la calidad del texto traducido, que puedan hacer uso de herramientas de apoyo a la traducción de forma razonada, que puedan discernir entre información fidedigna e información dudosa, y que puedan diferenciar un error de traducción de una intervención traductora a favor de la informatividad del texto o entendimiento del destinatario.

Se aprende a traducir haciéndolo de manera reflexiva, por ende, la didáctica de la traducción no debe centrarse solo en «lea, traduzca y pasemos a la revisión grupal», sino debe orientarse al fortalecimiento de actividades fundamentales, el tratamiento controlado de problemas y el análisis del error. Los textos para trabajar en clase deben seleccionarse en torno a los problemas que estos presentan para que el estudiante que culmine su formación en traducción cuente con un cúmulo de estrategias que pueda aplicar en la traducción de textos de diferentes géneros, campos y contextos. En la práctica docente, se observó que, en algunos casos, los problemas eran solucionados de forma automática sin mayor reflexión ni documentación. Los criterios para solucionar y evaluar traducciones eran muchas veces subjetivos. Cuando se pedía a los estudiantes que explicaran cómo llegaron a plantear sus alternativas traductoras, hacían referencia a diccionarios o glosarios. Eran pocos los que relacionaban sus propuestas con procedimientos inferenciales o diferentes tipos de análisis. Al pedirles que identificaran los errores cometidos, centraban 
su evaluación en cuestiones formales, y pocos identificaron errores que afectaban la transferencia del sentido. En cuanto a la autoevaluación, estaban más pendientes de cuánto podían afectar los errores a sus calificaciones que del progreso en la adquisición de la competencia estratégica.

En vista de esta situación, se propuso un programa de intervención centrado en la formación de pensadores críticos y el desarrollo de la competencia estratégica. Para ello, se realizó una selección de textos originales que presentaran problemas de diferente índole, a saber, textuales, pragmáticos y culturales, y se diseñaron actividades de análisis textual, análisis semántico, situacional y del error, así como ejercicios de traducción que requerían seguir un proceso controlado de resolución de problemas con la finalidad que los estudiantes mejoren la capacidad de solucionar problemas y evaluar la calidad de las traducciones propias y de terceros desde un punto de vista objetivo. El fin de nuestra investigación es determinar la influencia de un programa para el desarrollo del pensamiento crítico en la adquisición de la competencia estratégica de los estudiantes del primer taller de traducción.

\section{MarCo teórico}

\subsection{La competencia estratégica}

La competencia estratégica es el componente central de la macrocompetencia traductora e integra las competencias comunicativa-textual, cultural, instrumental, psicofisiológica, temática e interpersonal, la misma que se pone de manifiesto en el proceso de traducción (PACTE, 2005). El grupo de investigación sobre adquisición de competencia traductora, señala que la competencia estratégica es la más importante, ya que es la responsable de resolver los problemas y de la eficiencia del proceso traductor, lo que se logra planificando el proceso de traducción, evaluando el proceso en sí y los resultados parciales obtenidos. Asimismo, esta competencia permite compensar deficiencias al identificar los problemas de traducción y aplicar procedimientos para resolverlos.

El objetivo del primer taller de traducción es que los estudiantes interioricen el método profesional de traducir, adquieran competencia estratégica aplicando postulados teóricos a la práctica traductora, y empleen diversos procedimientos y técnicas de traducción para que puedan inferir normas operativas a partir del contexto y encargo de la traducción. La Rocca (2007) señala que el taller de traducción tiene como objetivo que los estudiantes adquieran la capacidad de redactar diferentes versiones para los textos propuestos 
y seleccionar la traducción más adecuada de acuerdo con diferentes criterios, siendo el principal la función de la traducción. Por su parte, Lazo y Zachary (2008) sostienen que la formación de traductores debe orientarse a preparar traductores/investigadores capaces de comprender el texto en su dimensión lógica e interdisciplinaria y entrenar a los traductores en un sistema de trabajo eficiente que permita la aplicación de conocimientos y competencias adquiridas para resolver cualquier tipo de problemas que se presentaran en su vida profesional. En ambos casos, estos autores hacen referencia a dimensiones del pensamiento crítico como la capacidad de tomar decisiones valorativas y solucionar problemas de forma creativa. Vienne (como se citó en Golcher, 2004) señala que analizar varias situaciones trasnacionales, así como evaluar y hacer uso de los recursos necesarios para el trabajo de traducción son capacidades que se relacionan directamente con el pensamiento crítico. Para realizar una traducción adecuada, se requiere haber desarrollado previamente la capacidad de investigar, analizar y razonar, así como de ser inquisitivo y juicioso, es decir todo lo que implica tener pensamiento crítico. En este contexto, Rojas (2004) señala que es deber del docente guiar a sus estudiantes en el desarrollo de la competencia traductora a través de una serie de actividades que permita experimentar aciertos y desaciertos, mediante estrategias de ensayo y error.

\subsection{El pensamiento crítico}

Elder y Paul (1994) definieron el pensamiento crítico como «el proceso intelectualmente disciplinado de conceptualizar, aplicar, analizar, sintetizar y/o evaluar de manera activa y diestra, información reunida de, o generada por, la experiencia, reflexión, razonamiento o comunicación, como guía para la creencia y la acción» (p. 34). En este caso, se hace referencia a habilidades vinculadas con la gestión de la información, así como la disposición a usar estas habilidades de forma efectiva. En el caso de la traducción, la comprensión de un texto implica cuestionarse sobre lo que dice y quiere decir el autor, y monitorear el proceso de comprensión a través de preguntas como ¿rrealmente el texto dice esto?, ¿existen pistas que avalen lo que estoy infiriendo?, ¡esta idea se relaciona con otras ideas del texto?, ¿estoy comprendiendo bien o estoy yendo más allá de lo que dice el texto?, entre otras. El traductor tiene que pensar desde la perspectiva del autor del texto original que fue producido en otro espacio, tiempo, lengua y cuyas normas socioculturales pueden diferir de las suyas. A la vez, tiene que pensar en cómo el destinatario va a entender el mensaje e intervenir en función de las diferencias culturales y el distanciamiento espacial-temporal. 
El traductor como pensador crítico debe ser consciente de sus limitaciones para acceder al conocimiento, a diferencia de un lector que lee y comprende textos de su especialidad, el traductor se enfrenta a textos de diferentes campos y registros. No es un especialista en todos los temas que traduce, ni conoce todos los géneros ni la terminología; por lo tanto, necesitará consultar a especialistas de otros campos u otros recursos documentales. Marciales (2003) sostiene que los pensadores críticos desarrollan humildad intelectual y reconocen los límites de su conocimiento, indagan sobre generalizaciones y ponen a prueba posibles excepciones, evalúan la credibilidad de las fuentes de información, porque reconocen la importancia de usar fuentes confiables; por ello, analizan fuentes alternativas y reúnen información adicional cuando encuentran alguna contradicción. Leen con un alto grado de escepticismo, pues saben que todos podemos cometer errores y dejar de lado información relevante, y consideran los cuestionamientos como una oportunidad para desarrollar su línea de pensamiento e intercambiar puntos de vista.

El pensamiento crítico usa destrezas para el manejo de la información y se opone a la adquisición superficial de información sin valorarla. Para Halpern (como se citó en Vásquez, 2010), el pensamiento crítico permite entender la forma en que

una causa es determinada, el reconocimiento y crítica de los supuestos, el análisis de relaciones entre medios y fines, la evaluación de grados de probabilidad e incertidumbre, el reconocimiento de problemas, la incorporación de datos aislados, en un marco amplio (p. 28).

El pensador crítico busca la verdad y no se deja llevar por la subjetividad. Uno de los problemas frecuentes en la revisión de una traducción es la falta de objetividad. Por ello, Gouadec (1989) propuso el uso de parámetros de revisión que facilite el análisis, codificación y estimación del impacto del error. De esta forma podría realizarse una evaluación ponderada de la calidad de la traducción en función de las dificultades del texto origen. Brunette (1998) sostiene que, para desarrollar la capacidad de revisión y auto revisión, el traductor-revisor debe evaluar si la traducción mantiene la continuidad del sentido, respeta la intención del autor y produce el efecto esperado. Asimismo, si se han analizado los elementos extralingüísticos que rodean la producción y recepción del texto, la norma lingüística y el uso de la lengua. Por su parte, Mossop (2001) plantea doce parámetros para revisar las traducciones divididas en cuatro grupos: transferencia, contenido, lenguaje y presentación. El parámetro de transferencia permite verificar si la traducción cumple su función, si el sentido es el mismo que el del texto origen, si se ha transmitido 
la totalidad del mensaje que contiene el texto origen y si no se han realizado adiciones ni omisiones innecesarias. El parámetro de contenido está relacionado con la lógica del texto, los datos y la terminología y fraseología utilizados por el traductor. El parámetro de lenguaje considera la norma, el uso de la lengua de llegada y la adecuación al destinatario de la traducción. El revisor debe asegurarse que los enunciados sean claros y concisos y si las expresiones y colocaciones son idiomáticas. El parámetro presentación se centra en verificar que no se haya omitido ningún párrafo y que la disposición textual y la ortotipografía sea adecuada. La utilización de estos parámetros dependerá del encargo de traducción, quienes son los destinatarios y la función del texto meta, así como si la revisión es parcial o completa. Antes de iniciar la revisión, el traductor debe preguntarse: ¿se van a aplicar todos los parámetros?, ¿en qué orden?, ¿se deben verificar todos los parámetros de forma conjunta o por separado?

\section{Metodología}

Esta investigación es aplicada, ya que tiene como objetivo la evaluación de un programa de intervención para el desarrollo del pensamiento crítico. El diseño de investigación es cuasiexperimental tomando como referencia el diseño con preprueba y posprueba con grupos no equivalentes (no aleatorizados), previamente conformados. Se aplicó la preprueba al grupo de control y al experimental para medir la competencia estratégica inicial. Una vez culminado el programa de intervención, se tomó una posprueba para determinar si había mejorado la competencia estratégica en el grupo experimental El programa de intervención tenía como objetivo estimular el desarrollo del pensamiento crítico especialmente en lo que se refiere a operaciones cognitivas de orden superior como el análisis, inferencia, interpretación, evaluación y autoevaluación. Se diseñó un portafolio de aprendizaje con veinte actividades adicionales a los encargos de traducción, dividida en cuatro bloques: actividades para desarrollar la capacidad de análisis, actividades para desarrollar la capacidad de inferencia, actividades para desarrollar la capacidad de evaluación y actividades para desarrollar la capacidad de autoevaluación. Se llevaron a cabo veinte sesiones de dos horas académicas, con una frecuencia de tres veces por semana a lo largo del semestre académico 2015-II. Se programaron actividades fuera del aula como las de documentación y análisis; las de evaluación y debate se realizaron en el aula. Las sesiones fueron dirigidas por el investigador, docente responsable del taller. 
El grupo experimental realizó ejercicios y actividades cognitivas de análisis textual, análisis componencial y de las relaciones de inclusión para seleccionar equivalentes adecuados. Asimismo, se ejercitó en la recuperación de información y actividades de documentación para el tratamiento de modismos, presuposiciones e implicaturas. Se trabajaron actividades metacognitivas al evaluar las traducciones de sus compañeros y sus propias producciones. El grupo de control recibió la enseñanza con el método tradicional: información extra textual, traducción y revisión grupal de traducciones. Tanto el grupo experimental (GE) como el grupo de control (GC) estuvieron conformados por 20 estudiantes regulares del primer taller de traducción inglés/español. El desarrollo de la capacidad de análisis se complementó con el desarrollo de la capacidad de inferencia. Esta capacidad fue fundamental para la captación del sentido del texto. Al igual que la capacidad de análisis, la inferencia requiere la ejercitación de la capacidad de observación e identificación de elementos presentes en el texto y de los conocimientos previos. Identificar el tema y el propósito del autor, así como el análisis de la situación comunicativa, permitirán al traductor lector captar el sentido general de las ideas, así como identificar las pistas que le proporciona el autor y los elementos verbales y no verbales que le permiten formular sus propias hipótesis de sentido y construir significados del texto. Para ejercitar estas capacidades, se aprovecharon todos los momentos y situaciones de aprendizaje posible, se seleccionaron textos de diversas temáticas y complejidades, se utilizó la interrogación didáctica para verificar la correcta captación del sentido y el reconocimiento de elementos implícitos. Se convirtió el aula en una comunidad de investigación definida por Lipman (como se citó en Serrano, 2008) como un entorno en que

los estudiantes se escuchan los unos a los otros con respeto, construyen sus ideas sobre las de los demás, se retan los unos a los otros para reforzar argumentos de opiniones poco fundadas, se apoyan en los procesos inferenciales a partir de lo que afirman y de sus elaboraciones (p.512).

El instrumento de recolección de datos, preprueba y posprueba fue validado por juicio de expertos. Ambas pruebas tuvieron la misma estructura, lo que varió fueron los textos empleados. Se cuidó que la extensión fuese aproximada. En el primer caso, 231 palabras y en el segundo, 234. La prueba se tomó en tres sesiones diferentes, cada una de 1:30 horas de duración. Cada prueba tenía tres partes correspondientes a tres dimensiones: solución de problemas, evaluación de traducciones y autoevaluación de traducciones, como se detalla a continuación. 
Figura 1. Estructura de la prueba
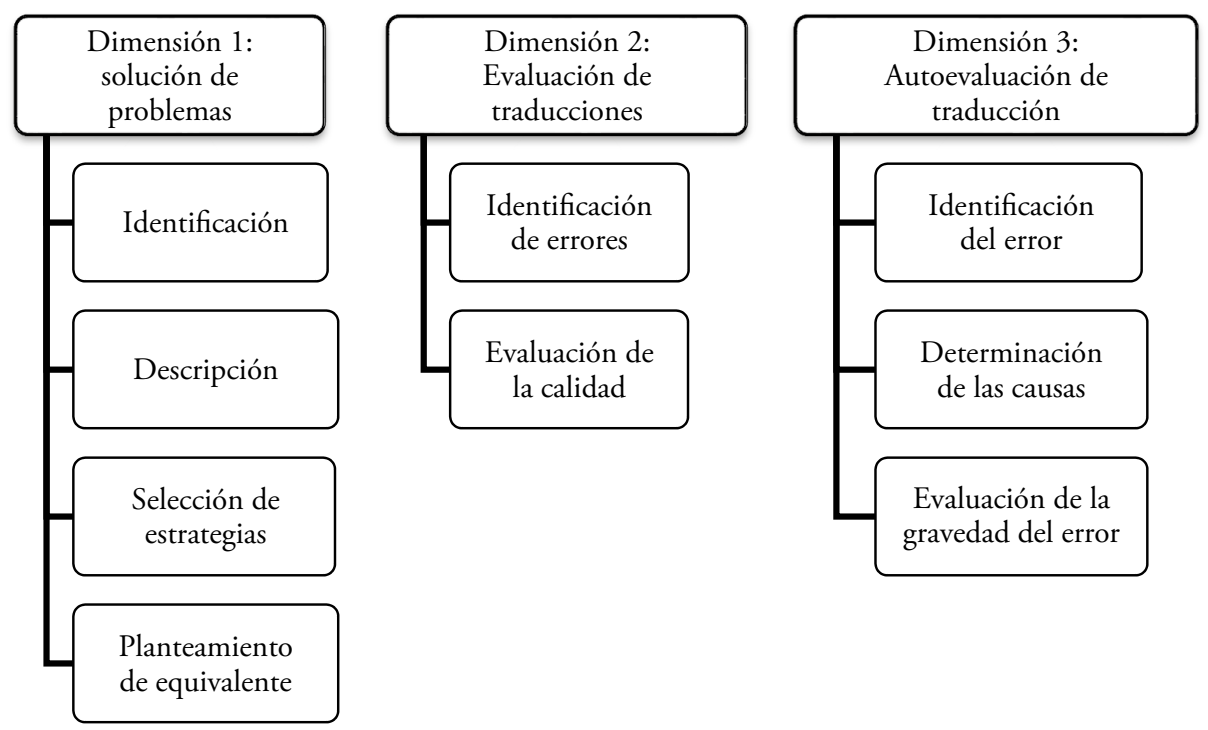

En la primera parte de ambas pruebas, se proporcionó un texto periodístico en inglés que presentaba quince problemas de traducción (juegos de palabras, vacíos denominativos, referentes culturales, unidades fraseológicas, polisemia y connotaciones), los mismos que debían ser identificados y descritos, luego se debería indicar la estrategia empleada para solucionarlo y, finalmente, proponer un equivalente adecuado como solución. En la segunda parte se entregaron textos originales con sus respectivas traducciones para que los estudiantes identificaran los errores según los parámetros de transferencia, contenido, lenguaje y presentación y evaluaran la calidad de la traducción, el impacto del error y estimaran la calidad de la traducción. Asimismo, se les entregó la traducción realizada en la primera parte para que detecten los errores cometidos, determinen la causa del error y estimen la gravedad del error en un informe de evaluación de la calidad de la traducción en tres párrafos. Este informe debía considerar la evidencia (registro de errores) y la conclusión (impacto del error en la calidad de la traducción). Se elaboró una rúbrica de evaluación del informe que consideraba cuatro aspectos: vocabulario, uso del metalenguaje de la traducción; redacción, evidencias y ejemplos de los errores cometidos; y conclusión o valoración de la calidad de la traducción a partir de la evidencia y los parámetros evaluados. En la tercera parte, se entregó a los estudiantes, la traducción realizada en la parte uno para que realicen el mismo 
procedimiento indicado en la segunda parte, más una actividad metacognitiva y reflexiva que consistía en identificar la causa de su error.

La información obtenida de las pruebas se procesó teniendo en cuenta las rúbricas para el análisis cuantitativo y cualitativo. El análisis de la posprueba es el punto central de la investigación, porque permite la verificación de la hipótesis: un programa de intervención para el desarrollo del pensamiento crítico tendrá un efecto significativo en la adquisición de la competencia estratégica de los estudiantes del primer taller de traducción. Los datos recogidos se procesaron en tablas de frecuencia y se aplicó la prueba $\mathrm{Z}$ para calcular la eficacia del programa de intervención en el desarrollo de las capacidades de:

- Resolver problemas: comprende una mejora en la capacidad de identificación de problemas, descripción de problemas, selección de estrategias y planteamiento de equivalentes para los problemas de traducción.

- Evaluar la calidad de las traducciones: comprende la capacidad de identificar errores por parámetro de revisión y capacidad de argumentación al estimar la calidad de una traducción.

- Autoevaluar la propia traducción: comprende la capacidad de identificar errores, determinar sus causas y estimar la gravedad del error.

\section{Resultados}

\subsection{Resolución de problemas}

Se establecieron escalas de medición para evaluar la capacidad de identificar problemas. El nivel avanzado se ubica entre 76 y $100 \%$ de problemas identificados; intermedio, entre 51 y $75 \%$; bajo, entre 26 y 50\%; y deficiente, por debajo del $25 \%$. El grupo experimental (GE) alcanzó un 50\% en el nivel avanzado. En cuanto a la descripción, el GE alcanzó un 50\% en el nivel avanzado, ya que describió claramente el tipo de problema. Con respecto al uso de estrategias, el GE empleó mayormente (35,5\%) estrategias como analizar la estructura de la oración, hacer un análisis semántico, hacer inferencias, revisar predicciones y manipular pragmáticamente el texto a diferencia del GC que recurrió a estrategias convencionales como documentarse por internet, consultar diccionarios y traducción literal $(44,3 \%)$. Tal como se observa en la Figura 2, el grupo experimental tuvo una mejor actuación: 30\% de diferencia en la identificación de problemas, $40 \%$ en la descripción de problemas, $27,17 \%$ en la selección de estrategias y $55 \%$ en el planteamiento del equivalente adecuado. 
Figura 2. Capacidad de resolver problemas

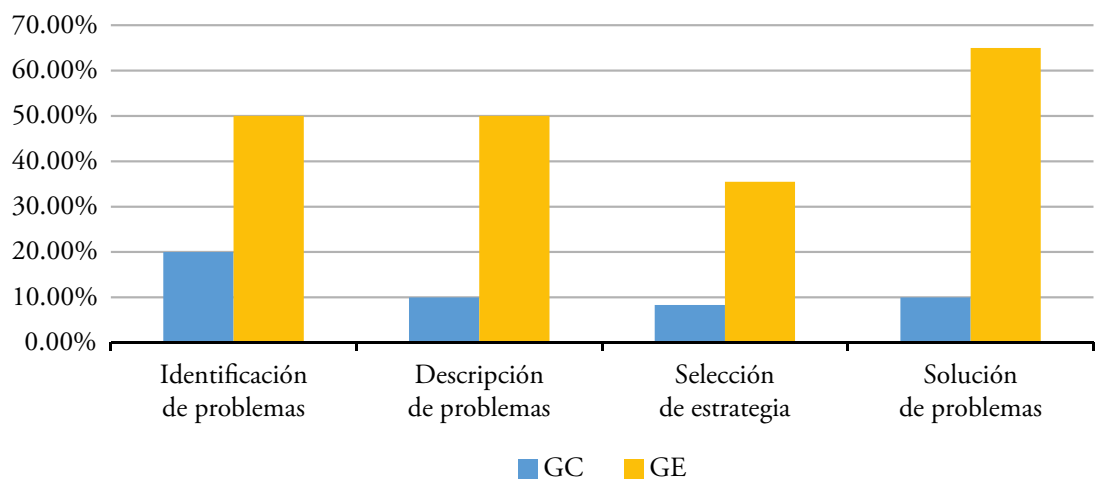

Fuente: Resultados de la posprueba.

\subsection{Evaluación de traducciones}

El texto seleccionado para evaluar la calidad de la traducción presentaba errores en todos los parámetros. En la Tabla 1, se presentan los errores existentes y los identificados por grupo.

Tabla 1. Errores existentes en la traducción y promedio de errores identificado por grupo

\begin{tabular}{llccc}
\hline \multicolumn{1}{c}{ Parámetro } & \multicolumn{1}{c}{ Indicador } & Existente & GC & GE \\
\hline \multirow{2}{*}{ Transferencia } & Falso sentido & 5 & 1,9 & 4,75 \\
& Adición & 1 & 0,2 & 0,9 \\
& Omisión & 3 & 0,8 & 2,8 \\
\hline Contenido & Falta coherencia & 2 & 0,6 & 1,5 \\
& Expresión incongruente & 1 & 0,55 & 0,06 \\
& Error de selección lexical & 8 & 1,7 & 5,25 \\
\hline \multirow{2}{*}{ Lenguaje } & Preposiciones inadecuadas & 1 & 0,4 & 1 \\
& Uso inadecuado de artículos & 1 & 0,65 & 0,8 \\
& Calco & 9 & 1,3 & 7,75 \\
& Gerundio & 1 & 0,6 & 1 \\
& Sintaxis & 1 & 0,8 & 1 \\
\hline \multirow{2}{*}{ Presentación } & Deletreo incorrecto & 1 & 0,95 & 0,95 \\
& Tildes & 2 & 1,4 & 1,4 \\
& Cursivas, negritas, versalitas & 6 & 1,25 & 5,8 \\
& Signos de puntuación & 3 & 1,95 & 2,55 \\
\hline
\end{tabular}


Como se aprecia en la Figura 3, el GE identificó más errores en los cuatro parámetros, con una diferencia significativa en transferencia $(61,67 \%)$, contenido $(36 \%)$, lenguaje $(60 \%)$ y presentación $(42,92 \%)$.

\section{Figura 3. Identificación de errores}

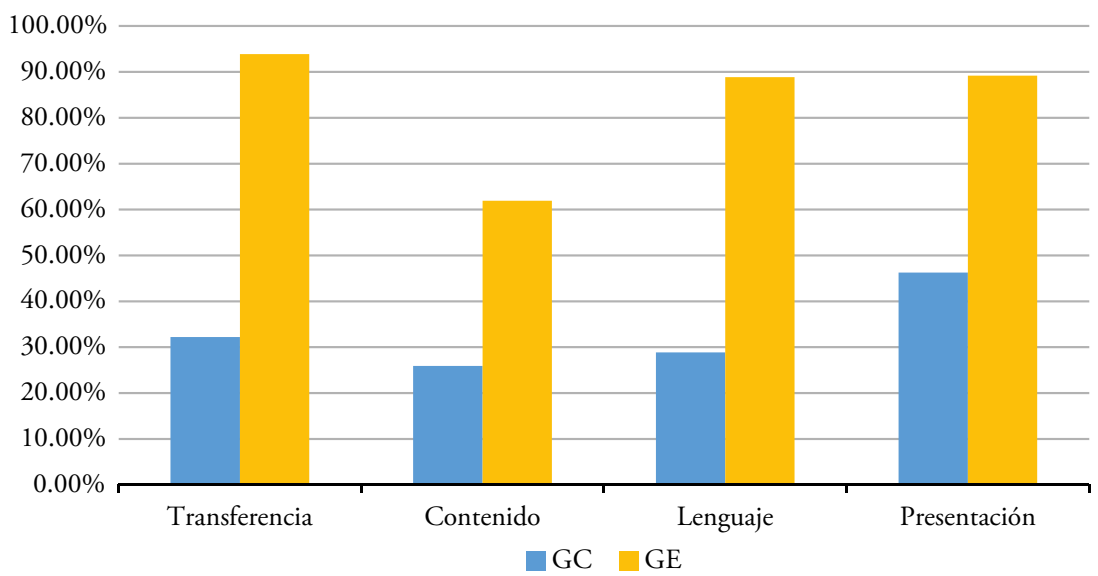

Fuente: Resultados de la posprueba.

El registro de identificación de errores fue el insumo para realizar el informe de evaluación de la calidad de la traducción. Para ello, se tomó en consideración los siguientes rubros: vocabulario, redacción, evidencia y conclusión. El GE alcanzó un nivel intermedio en el uso de vocabulario y la capacidad de redacción, asimismo, un nivel avanzado en evidencia y conclusión. El rubro de vocabulario estaba asociado con el uso del metalenguaje de la traducción y era un indicador de la internalización de nociones básicas (como equivalencia, fidelidad, intencionalidad, textualidad, informatividad, situacionalidad), que permite que la evaluación sea objetiva. En cuanto a la redacción, si bien el GE no alcanzó el nivel avanzado, se evidenció un progreso en la articulación lógica de las oraciones a pesar de algunos errores de cohesión. Los resultados óptimos en evidencia y conclusión se derivan de la buena actividad de identificación de errores. El trabajar con parámetros permitió evaluar como estos errores afectan la calidad de la traducción. Los resultados presentados en la Figura 4 muestran un mejor desempeño del GE con diferencias significativas de $45 \%$ en uso de vocabulario, $45 \%$ en redacción, $70 \%$ en evidencia y $70 \%$ en conclusión. 
Figura 4. Informe de evaluación de la calidad de la traducción

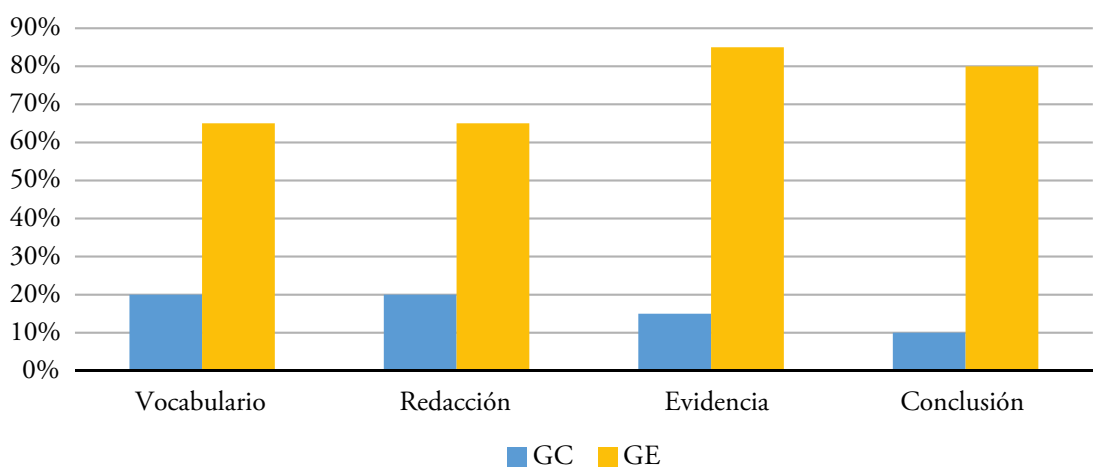

Fuente: elaboración propia con los datos de las pruebas.

\subsection{Autoevaluación de traducciones}

Para medir la capacidad de autoevaluación se empleó la prueba de traducción que rindieron para solucionar los problemas de traducción. Se revisó cada una de las pruebas rendidas por los estudiantes del GC y GE, se vaciaron en una matriz y se sacó el promedio de errores cometidos por grupo. Esta cifra nos permitió contrastar los errores cometidos y los identificados, la misma que se presentan en la Tabla 2

Tabla 2. Número y porcentaje de errores cometidos e identificados por el GC y GE

\begin{tabular}{lcccccc}
\hline & \multicolumn{3}{c}{ Grupo de control } & \multicolumn{3}{c}{ Grupo experimental } \\
\cline { 2 - 7 } & Identificado & Cometido & $\%$ & Identificado & Cometido & $\%$ \\
\hline Transferencia & 21 & 90 & 23,33 & 39 & 65 & 60,00 \\
Contenido & 26 & 96 & 27,08 & 43 & 62 & 69,35 \\
Lenguaje & 55 & 165 & 33,33 & 32 & 55 & 58,18 \\
Presentación & 48 & 90 & 53,33 & 29 & 33 & 87.87 \\
Total & 150 & 376 & 39,89 & 143 & 215 & 66,51 \\
\hline
\end{tabular}

Fuente: Elaboración propia con los datos de la posprueba.

Los resultados que se presentan en la Figura 5 muestran las diferencias de ambos grupos en los cuatro parámetros: $36,67 \%$ en la identificación de errores de transferencia, $40,24 \%$ en la identificación de errores de contenido, 24,85\% en la identificación de errores de lenguaje y $35,54 \%$ en la identificación de errores de presentación. 
Figura 5. Identificación de errores propios

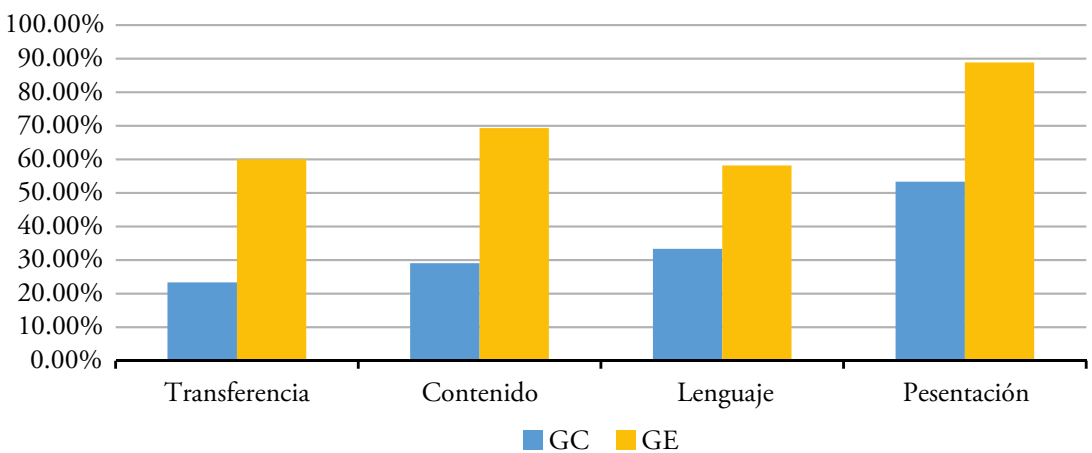

Fuente: Datos de la posprueba.

Ambos grupos señalaron que las causas de error se debían a malas actuaciones vinculadas con la aplicación de estrategias y procedimientos de traducción, además de otros factores vinculados con la concentración y planificación. El GC identificó como causas de errores más recurrentes la falta de tiempo y el apresuramiento, lo que evidencia falta de organización y planificación. Los resultados de la capacidad de argumentación en el informe de evaluación de la calidad de su traducción se presentan en la Figura 6, en la que se aprecia un mejor desempeño del GE en las dimensiones vocabulario (60\%), redacción $(60 \%)$, evidencia $(65 \%)$ y conclusión $(70 \%)$.

Figura 6. Informe de evaluación de la calidad de su traducción

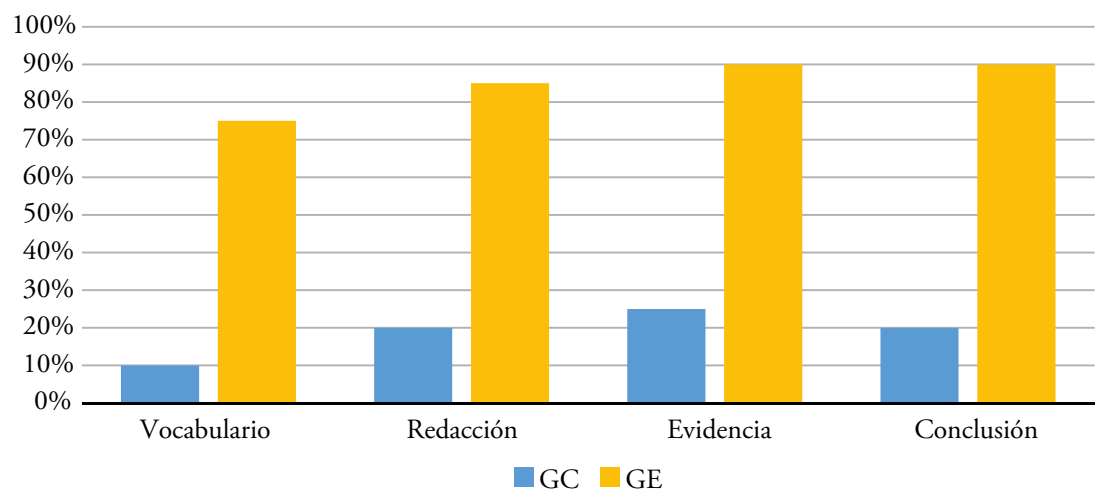

Fuente: Datos de las pruebas. 


\section{Discusión}

Los resultados obtenidos nos permiten deducir que un programa de intervención para el desarrollo del pensamiento crítico basado en actividades cognitivas y metacognitivas favorece la adquisición de la competencia estratégica. La exposición a una metodología que propicia el aprendizaje basado en el desarrollo de operaciones cognitivas para solucionar problemas de comprensión y expresión demostró ser adecuada para el desarrollo de la competencia estratégica. El 65\% de acierto en el planteamiento de equivalentes para solucionar los problemas textuales, pragmáticos y culturales confirmó lo establecido por Betancourth, Enríquez y Castillo (2013) en relación con la capacidad de análisis o la descomposición de un evento, argumento o situación en sus partes para establecer comparaciones y relaciones entre las partes que lo componen. En el programa de intervención, se ejercitó el análisis de relaciones de inclusión, así como de semas comunes y diferenciadores para establecer equivalentes entre términos aparentemente sinónimos que aparecían en un mismo texto como stepfamily/blended family/second marriage fammily y overparenting/ overprotection/ overinvolvement/ overcomfort, overindulge. En el primer caso, un gran porcentaje de la clase no estableció diferencias entre los tres términos, ya que en las fuentes y herramientas de documentación aparecían como sinónimos, pero luego del análisis de la situación comunicativa empezaron a establecer equivalencias creativas incluso frases que transmitían el sentido y connotación particular de cada uno de los términos. En el segundo caso, se trató de no caer en el préstamo ni el calco y buscar equivalentes que marcaran una diferencia clara entre los términos, asimismo, los estudiantes entendieron que tenían que usar recursos morfológicos diferentes en español, al no tener nuestra lengua la flexibilidad que tiene el inglés para la formación de palabras compuestas y el recurso de la yuxtaposición. Una vez que el alumno ha desarrollado el pensamiento crítico puede hacer uso más efectivo de operaciones cognitivas superiores como la inferencia, la deducción e inducción. El entrenamiento permitió que los del grupo experimental pudieran plantear equivalentes creativos y comunicativos para términos que aparecieron en el texto de salida como free range childhood.

Nuestro programa inició priorizando la capacidad de análisis tanto en su vertiente formalizada por modelos de análisis lingüísticos, textuales, discursivos y situacionales como otros de naturaleza espontánea y creativa. Desde el punto de vista cognitivo, la capacidad de análisis implica los siguientes procesos mentales: observar, relacionar, comparar, discriminar, seleccionar, interpretar e integrar, capacidades que fueron desarrolladas de forma integral 
y que permitieron solucionar diferentes tipos de problemas como el de selección lexical. Los problemas de selección lexical suelen ser los más complejos y recurrentes para los estudiantes de traducción. Machado (2012) afirma que el traductor prioriza las correspondencias léxicas, al confrontar la traducción con el texto original y la correspondencia semántica en relación con la adecuación a la situación meta y el uso en el contexto de llegada. "Este profesional se mueve por caminos «movedizos», haciendo un puente entre textos, transportando de una determinada lengua para otra, vocablos con sus sentidos y significados, siempre pensando en el contexto» (p. 4). Por lo tanto, se requería ejercitar la capacidad de observación y discriminación por medio del análisis semántico, componencial y contextual. Se puso en marcha un programa de base cognitivo y metacognitivo para limitar la dependencia al diccionario bilingüe y apostar por el análisis de las marcas de coherencia y cohesión, los principios de selección y jerarquía de las teorías funcionalistas de la traducción para establecer el principio de adecuación léxica, teniendo en cuenta el contexto, cotexto y el propósito del texto. En este sentido, el análisis del texto original tuvo un impacto positivo a pesar de la resistencia inicial de los estudiantes del grupo experimental. Existía una tendencia a la inmediatez por traducir (trasladar). El análisis no les resultaba atractivo y tomaba mucho tiempo. No obstante, a medida que se desarrollaba el programa entendieron la necesidad de atravesar por este proceso para obtener una traducción precisa, sin dudas y ambigüedades.

Según Machado (2012), la conciencia léxico-semántica monitorea la selección lexical adecuada en base a la carga semántica de la palabra y su adecuación al contexto, ya que las unidades lexicales con significados muy amplios pueden ocasionar problemas de ambigüedad. Por lo tanto, se emplearon modelos de análisis que permitieran el acceso a redes léxico-semántico de vocablos más adecuados al texto y contexto. De esta forma se solucionaron problemas de «falsos amigos», ambigüedades y fronteras semánticas vagas. Se observó que la adecuación léxico-semántica, en relación con el contexto, es una herramienta que permite tomar conciencia del valor semántico de la unidad léxica en el plano denotativo, connotativo y pragmático. Esto confirma lo expresado por Machado (2012) al sostener que «el léxico y su carga semántica, adecuados al contexto, son componentes básicos para el proceso de traducción, y la selección de estos componentes, de forma correcta, es fundamental para que se llegue a una traducción eficaz y comprometida» (p. 5).

De esta forma y a través del análisis continuo, se minimizaron los errores de selección léxica y fraseológica que, según señala La Rocca (2007), se pueden atribuir a la falta de competencia de documentación y/o a la capacidad para 
inferir el significado en base a conocimientos previos o «a un insuficiente balance entre procesos ascendentes y descendentes de comprensión; a una falta de competencia en la aplicación de estrategias de búsqueda de equivalencias, reformulación y verificación en el caso de la fase de reexpresión» (p. 187). Por lo tanto, se requiere fomentar el entrenamiento estratégico cognitivo centrado en el abordaje de problemas que atañen tanto a la fase de comprensión como de expresión. Los resultados de las preprueba y posprueba muestran diferencias significativas entre el desarrollo de la capacidad de análisis e inferencia. Estas capacidades se trabajaron de forma conjunta durante la intervención, y permitieron a los estudiantes del grupo experimental deducir hipótesis de sentido y realizar afirmaciones razonables. La capacidad de evaluación también fue transversal al programa de intervención, ya que no puede disociarse de la capacidad de analizar e inferir y, como bien señala Golcher (2004), está integrada en las estrategias cognoscitivas para evaluar la credibilidad de las fuentes de información, analizar argumentos, evaluar soluciones, examinar o evaluar suposiciones, evaluar evidencia y presuntos hechos.

El desarrollo de estas capacidades está estrechamente relacionado con la solución de problemas, lo que confirma el impacto que tuvo el programa de intervención en los puntajes obtenidos por los estudiantes del grupo experimental en sus cuatro dimensiones: identificación, descripción, aplicación de estrategias y propuesta de equivalente, las cuales son justamente los componentes del pensamiento crítico. Por consiguiente, se confirma lo que señala Golcher (2004), el traductor debe aclarar los problemas de traducción para identificar lo que necesita para resolverlos, con el fin de poder realizar un análisis de las posibles soluciones. El traductor crítico requiere aclarar el pensamiento para comprender los conceptos y argumentar objetivamente el porqué de la elección de una palabra, término o expresión en un contexto dado. El estudiante de traducción debe tener conciencia de los criterios que rigen la selección o descarte de un término o expresión mientras traduce, de esta forma sus elecciones traductoras responderán a una comprensión profunda.

En cuanto a la segunda dimensión de la competencia estratégica, evaluación de la calidad de la traducción, los estudiantes del grupo experimental lograron llegar a conclusiones bien razonadas de acuerdo con parámetros para medir la calidad. En un primer momento, se buscó que los estudiantes interiorizaran el concepto de calidad y la importancia de presentar trabajos y tareas de calidad. Se instauró en común acuerdo con los estudiantes del programa de intervención la noción de «traducción inaceptable». De esta forma, las versiones que no habían sido revisadas por los estudiantes no merecían ser evaluadas en clase, porque no cumplían con los estándares mínimos. Si un 
objetivo del programa era lograr autonomía del estudiante, esta tenía que construirse en base a la responsabilidad e integridad. Estrechamente vinculada con la competencia estratégica se encuentra la competencia psicofisiológica, que según Kelly (2002) comprende habilidades psicológicas necesarias para ser un traductor profesional (atención, memoria, confianza). El desarrollo del pensamiento crítico otorga mayor confianza a los estudiantes y permite la automatización de tareas de transferencia.

La tercera dimensión autoevaluación de traducciones está relacionada estrechamente con la capacidad de reflexión, la cual es una característica fundamental del pensamiento crítico y está vinculada con las operaciones metacognitivas que permite reflexionar sobre lo que están haciendo. En este sentido, apostamos por una pedagogía del error, entendiendo el error como un sensor de problemas, tal como lo afirma De la Torre (2004):

Es un indicador del proceso y no un resultado sancionable o punible. El marco que neutraliza la consideración del error es, naturalmente, el proceso de aprendizaje. Gracias al mismo podemos obtener información sobre los mecanismos mentales, que el acierto no nos proporciona (p. 8).

El error es el punto de partida para decodificar el proceso seguido e indagar las causas que intervinieron en el mismo.

Fomentar actividades metacognitivas resultó una tarea interesante, al igual que compartir las experiencias de ensayo y error, a través del diario del estudiante. El que los estudiantes tomen consciencia de cómo se trabaja y evalúen su proceso de adquisición de competencias fue interesante y motivador. Estas actividades permitieron que la clase sea más participativa, más analítica, los estudiantes se sintieron más libres para identificar los errores de sus compañeros, quienes a su vez defendieron sus versiones o reconocieron sus errores. En la prueba de salida, se observó un incremento en la identificación de errores de transferencia y contenido, las cuales están relacionados con una comprensión más profunda del texto. Por lo tanto, los puntajes obtenidos en la capacidad de evaluar y autoevaluar sus propias traducciones está estrechamente relacionado con una mejor adquisición de la competencia estratégica, lo que es coherente con lo que señala La Rocca (2007) sobre la importancia de la concienciación de los procesos cognitivos en la didáctica de la traducción: «el entrenamiento estratégico cognitivo y la reflexión metacognitiva realizada por los mismos estudiantes a través del análisis de sus propias traducciones son fundamentales para mejorar su competencia traductora» (p. 168). 


\section{Conclusiones}

El pensamiento crítico se puede desarrollar a través de la formulación de actividades que ejerciten operaciones cognitivas de orden superior, a saber, analizar, inferir, sintetizar e interpretar, y que integren a su vez actividades básicas como identificar, observar, clasificar, entre otras.

El programa de intervención tuvo una influencia significativa en la adquisición de la competencia estratégica en los estudiantes de Traducción. Las pruebas aplicadas y los resultados obtenidos permitieron confirmar nuestras hipótesis iniciales con respecto a la necesidad de diseñar un programa de intervención para el desarrollo del pensamiento crítico que comprendiese actividades cognitivas y metacognitivas.

El programa de intervención tuvo una influencia significativa en la capacidad de resolución de problemas del grupo experimental, tal como se evidencia en las diferencias porcentuales del grupo experimental con respecto al de control. $50 \%$ frente a $20 \%$ en la identificación, $50 \%$ frente a $10 \%$ en la descripción, $35,5 \%$ frente a $8,33 \%$ en la selección de estrategias y $65 \%$ frente a $10 \%$ en la solución de problemas (planteamiento de equivalentes).

El programa de intervención también tuvo una influencia significativa en la capacidad de evaluación de traducciones del grupo experimental, lo que se evidencia en la diferencia porcentual entre el grupo experimental y de control para identificar errores (en promedio 50\%) y para argumentar en el informe de evaluación (en promedio $57,5 \%$ )

El programa de intervención tuvo una influencia significativa en la capacidad de autoevaluar las traducciones del grupo experimental, lo que se evidencia en la diferencia porcentual entre el grupo experimental y de control para identificar errores propios (en promedio 34,25\%), para determinar la causa del error (en promedio 40,25\%) y para determinar la gravedad del error (en promedio $58,75 \%$ )

Frente al desafío que implica el desarrollo de la inteligencia artificial en sus dimensiones de rapidez y economía, el traductor podrá mantenerse en el mercado en tanto su habilidad de traducir vaya de la mano con la habilidad de revisar traducciones. Por lo tanto, el desarrollo del pensamiento crítico es fundamental para la realización de ambas tareas, sobre todo, para textos de mayor complejidad. 


\section{REFERENCIAS BIBLIOGRÁFICAS}

Betancourth, S., Enríquez, A. y Castillo, P. (2013). La controversia-socrática en el desarrollo del pensamiento crítico en estudiantes universitarios. Revista Virtual Universidad Católica del Norte, (39), 71-84. Recuperado de https:// www.redalyc.org/articulo.oa?id=1942/194227509007

De La Torre, S. (2004). Aprender de los errores: el tratamiento didáctico de los errores como estrategia de innovación. Recuperado de http://www.terras.edu.ar/ cursos/116/biblio/76Bases-teoricas-del-error.pdf

Brunette, L. (1998). La correction des traductions pédagogiques. En J. Delisle y H. Lee-Jahnke (Eds.), Enseignement de la traduction et traduction dans l'enseignement. Ottawa: Les Presses de l'Université d'Ottawa. Recuperado de http://books.openedition.org/uop/1826

Elder, L. y Paul, R. (1994). Critical Thinking: Why we must transform our teaching. Journal of Developmental Education, 18(1), 34-35.

Golcher, I. (2004). Educación del traductor como pensador crítico. Letras, (36), 65-80. Recuperado de http://www.revistas.una.ac.cr/index.php/letras/ article/viewFile/3617/3474

Gouadec, D. (1989). Comprendre, évaluer, prévenir: pratique, enseignement et recherche face à l'erreur et à la faute en traduction. TTR, 2(2), 35-54. https://doi.org/10.7202/037045ar

Kelly, D. (2002). Un modelo de competencia traductora: bases para el diseño curricular. Recuperado de http://wdb.ugr.es/-greti/revista-puentes/pub1/02Kelly.pdf

La Rocca, M. (2007). El taller de traducción: Una metodología didáctica integradora para la enseñanza universitaria de la Traducción (Tesis para optar el grado de doctora en Traducción). Universitat de Vic, Departamento de Traducción e Interpretación.

Lazo, M. y Zachary, M. (2008). La enseñanza de la traducción centrada en el estudiante. Onomázein, (17), 173-181. Recuperado de http://www.acuedi. org/doc/4233/la-enseanza-de-la-traduccin-centrada-en-el-estudiante.html

Machado, J. (2012). La selección léxica en traducción: aspectos semánticos y contextuales. Revista Cientifica Semana Académica. Recuperado de http://semanaacademica.org.br/artigo/la-seleccion-lexica-en-traduccionaspectos-semanticos-y-contextuales

Marciales, G. (2003). Pensamiento crítico: diferencias en estudiantes universitarios en el tipo de creencias, estrategias e inferencias en la lectura critica de textos (Tesis para optar el grado de doctor). Universidad Complutense de Madrid, Madrid. 
Morin, E. (1999). Los siete saberes necesarios a la educación del futuro. París: Unesco.

Mossop, B. (2001). Objective Translational Error and the Cultural Norm of Translation. TTR (Traduction, Terminologie, Rédaction), 2(2), 55-70. https://doi.org/10.7202/037046ar

PACTE (2005). Investigating translation competence: Conceptual and methodological issues. Meta, 50(2), 609-619. https://doi.org/10.7202/011004ar

Pineda, M. y Cerrón, A. (2015). Pensamiento crítico y rendimiento académico de estudiantes de la Facultad de Educación de la Universidad Nacional del Centro del Perú. Horizonte de la Ciencia, 5(8), 105-110. https://doi. org/10.26490/uncp.horizonteciencia.2015.8.126

Reupo, R. (2015). Propuesta de una estrategia didáctica, incorporando el uso de las TIC para mejorar el nivel de pensamiento crítico en estudiantes de ingeniería de sistemas, en el curso Cálculo diferencial, 2014 (Tesis para optar el grado de magíster en Informática Educativa y Comunicación). Universidad Católica Santo Toribio de Mogrovejo, Chiclayo.

Rojas, O. (2004). El portafolio y la evaluación en el proceso de traducción. Letras, 36, 1 (47). Recuperado de http://www.revistas.una.ac.cr/index.php/letras/ article/view/3616

Serrano, M. (2008). El desarrollo de la comprensión crítica en los estudiantes universitarios: hacia una propuesta didáctica. Educere: Revista Venezolana de Educación. Recuperado de https://bit.ly/2VTevKy

Vásquez, A. (2010). Competencias cognitivas en la educación superior. Revista Electrónica de Desarrollo de Competencias (REDEC), 6(2), Universidad de Talca. 